\section{REVISTA}

EDUGACIÓN
Revista Educación

ISSN: 0379-7082

ISSN: 2215-2644

revedu@gmail.com

Universidad de Costa Rica

Costa Rica

\title{
Las tecnologías computacionales y su repercusión en el proceso de formación matemática en la República de Angola
}

\author{
Faustino, Arnaldo; Wongo Gungula, Eurico; Arrocha Rodríguez, Olaysi \\ Las tecnologías computacionales y su repercusión en el proceso de formación matemática en la \\ República de Angola \\ Revista Educación, vol. 43, núm. 1, 2019 \\ Universidad de Costa Rica, Costa Rica \\ Disponible en: http://www.redalyc.org/articulo.oa? $\mathrm{id}=44057415016$ \\ DOI: https://doi.org/10.15517/revedu.v43i1.25502
}

Esta obra está bajo una Licencia Creative Commons Atribución-NoComercial-SinDerivar 3.0 Internacional. 


\section{Las tecnologías computacionales y su repercusión en el proceso de formación matemática en la República de Angola}

Computer technology and its impact on the process of mathematical formation in the Republic of Angola

Arnaldo Faustino *

Universidad Óscar Ribas (UÓR), Angola

arnaldo.faustino19@gmail.com

Eurico Wongo Gungula**

Universidad Óscar Ribas (UÓR), Angola

euricowongowongo@gmail.com

Olaysi Arrocha Rodriguez ${ }^{* * *}$

Universidad Máximo Gómez Báez (UNICA), Cuba

olaysiar@sma.unica.cu
DOI: https://doi.org/10.15517/revedu.v43i1.25502

Redalyc: http://www.redalyc.org/articulo.oa?id=44057415016

Recepción: 30 Junio 2016

Aprobación: 22 Noviembre 2018

\section{RESUMEN:}

Fortalecer la Institución de la Enseñanza Superior (IES), con la aplicación de las Tecnologías de la Información y la Comunicación (TIC), contribuye al perfeccionamiento del proceso de formación matemática, el cual impone la sistematización de los resultados investigativos para el estímulo del desarrollo científico, tecnológico, económico, social, cultural desde el año 2013 hasta el 2018 en las IES angoleñas, que restringen las potencialidades intelectuales, por las insuficiencias en interpretar la problemática en el ejercicio de la futura profesión. De ahí, se plantea como objetivo de la presente investigación: diagnosticar la situación actual del proceso de enseñanza-aprendizaje y la utilización de las tecnologías computacionales, a fin de minimizar las inconsistencias teóricas en la contextualización de los problemas y su significado. Los resultados de la pesquisa indican que es necesario promover eficazmente la utilización de las TIC y la pedagogía de la matemática en el aprendizaje a largo plazo, como un instrumento imprescindible para la transformación del país. La instrumentación de los resultados alcanzados en la pesquisa, permitió aplicar el enfoque cuali-cuantitativo, basado en la recopilación de los datos sobre la observación partícipe, la entrevista profunda y la aplicación sucesiva de los cuestionarios en las clases, lo cual también posibilitó triangular metodológicamente la información para indagar en las insuficiencias del proceso de enseñanza-aprendizaje de la matemática, por un sistema prediseñado de categorías, donde la participación comprometida del colectivo de los investigadores permitió recomendar y estimular el desarrollo de las habilidades necesarias para dinamizar los ejercicios de la futura profesión.

Palabras clave: Enseñanza-aprendizaje, pedagogía de la matemática, Tecnologías de la Información y la comunicación, Institución de la Enseña Superior, Recursos tecnológicos.

\section{Abstract:}

To strengthen the Institution of Higher Education (IHE), through the application of Information and Communication technology (ICT), which contributes to the improvement of the mathematical training process; research results are enforced to stimulate scientific, technological, economic, social, and cultural development from the year 2013 to 2018 in the Angolan IHE, and where intellectual potential is being restricted due to the failure to interpret problems with the profession. Hence, it is proposed as the objective of the present investigation; to diagnose the current situation of the teaching-learning process as well as the use of computer technologies, with the intent to minimize the inconsistencies in the contextualization of the problem and their meaning. Thus, the results of the research indicate that it is necessary to effectively promote the use of ICT and the pedagogy

\section{NoTAS DE AUTOR}

* ORCID: https://orcid.org/0000-0003-3197-5348

** ORCID: https://orcid.org/0000-0002-5685-1328

*** ORCID: https://orcid.org/0000-0003-3625-7353 
of mathematics in long-term learning, as an essential instrument for the transformation of the country. The results achieved in the research were obtained through the application of the qualitative-quantitative approach, in which data was collected by participant observation, in-depth interviews and questionnaires handed in classes. All of it made it possible to obtain the information necessary to investigate the insufficiencies in the teaching-learning process of Mathematics, by a pre-designed system of categories, where the committed participation of the group of researchers allowed to recommend and stimulate the development of the necessary skills to boost the exercises of the profession.

KEYWORDS: Teaching-learning, pedagogy of mathematics, Information and Communication Technology, Institution of Higher Education, Technological resources.

\section{INTRODUCCIÓN}

La posibilidad de alcanzar una estructura productiva basada en el desarrollo del conocimiento científico y tecnológico en la universidad, depende en gran parte del tiempo invertido en investigación y desde la perspectiva de la pertinencia en fortalecer sus capacidades en materia de ciencias vinculadas a una formación profesional, capaz de responder a los requerimientos de su entorno.

Autores como Martínez (1999) y Ramírez (2012) plantean la renovación del proceso de formación matemática, con la integración de las tecnologías computacionales en las IES como el componente decisorio en la formación, lo cual contribuye a dinamizar las investigaciones en función de las Políticas Nacionales de Ciencia Tecnológicas e Innovación (PNCTI) y la actitud positiva de los universitarios hacia las transformaciones del contexto social. Entendido este, como el entorno económico, político y sociocultural que envuelven a los futuros profesionales como sujetos históricos, sociales y culturales.

Actualmente en las IES públicas, público-privadas y privadas, en la sociedad angoleña, se observan, en la carrera de Licenciatura en Educación Matemática, limitaciones epistemológicas alarmantes en la aplicación de métodos activos y sistemas de procedimientos didácticos que imposibilitan la integración de las tecnologías computacionales, orientadas a lograr la motivación del estudiantado (futuros profesionales) en la dinámica del proceso de formación matemática, por tal razón es necesario que el Ministerio de la Enseñanza Superior Ciencia Tecnología e Innovación (MESCTI) implemente políticas para eliminar el éxodo de profesionales de excelencia en países desarrollados para reducir las deficiencias epistemológicas manifestadas en la dinámica del proceso de enseñanza-aprendizaje.

Por tanto, en aras de contribuir a minimizar las insuficiencias metodológicas en las IES públicas y privadas angoleñas, se diagnosticó la situación actual del proceso de formación matemática, con la utilización de las tecnologías computacionales, para minimizar las inconsistencias en la contextualización de los problemas y en la construcción de significados en el ejercicio profesional como un elemento importante en el desarrollo regional y nacional.

\section{RefERENTES CONCEPTUALES}

¿Qué hacer con las tecnologías computacionales en la dinámica del proceso de formación matemática angoleña para superar las limitaciones epistemológicas? Actualmente, la mejora sustentabilidad de las condiciones de vida de los angoleños, el combate a la corrupción en todos los sectores, la reducción de la desigualdad, la pobreza y un manejo adecuado de los recursos naturales son objetivos que difícilmente se pueden alcanzar dentro de los estrechos márgenes de las actuales estrategias de desarrollo, poniendo énfasis en la necesidad de desarrollar un proceso de formación investigativa sustentado en las TIC, en relación con el contexto histórico, social, cultural y universal en función de lograr su pertinencia social de acuerdo con la Estrategia Nacional de Ciencia Tecnología e Innovación (ENCTI) del país (Angola Pró-Reitoria para Reforma Curricular, 2007). La formación investigativa en el presente artículo es entendida como, el proceso de sistematización de los conocimientos y métodos de la investigación científica para solucionar los problemas 
de manera independiente, aplicando las tecnologías computacionales, mediante la apropiación de la lógica profesional, la cual convierte a los futuros profesionales en transformadores de la realidad en que vive apoyado en la innovación tecnológica de alto nivel.

A pesar de los adelantos de la ciencia y la técnica en el mundo y el desarrollo del pensamiento-investigativo, aún se necesita fomentar en los futuros profesionales la capacidad de emplear conceptos, representaciones y procedimientos abstractos secuenciales en la formación investigativa para entender las situaciones del mundo real, tanto en los aspectos de la vida como en el ámbito laboral.

La formación investigativa en el alumnado requiere del proceso de apropiación de perdurables conocimientos, a fin de desarrollar un pensamiento científico, reflexivo, crítico, estratégico e innovador que les posibilite integrar las tecnologías computacionales en la dinámica del proceso de formación matemática, bajo un paradigma alternativo sobre su función estratégica hacia un desarrollo con nuevas prioridades apoyadas en las políticas diseñadas para dinamizar el Paquete de Ciencia y Tecnología (PCT) para el siglo XXI. La dinámica del proceso de formación matemática es entendida "como todo el proceso de enseñanzaaprendizaje de la matemática, donde los futuros profesionales adquieren los contenidos estrechamente vinculados con los métodos de la investigación científica" (Faustino, Wongo y Craib, 2015a, p. 576) para enfrentar los desafíos del nuevo siglo y superar las limitaciones en la formación matemática.

Las tecnologías computacionales en las IES angoleñas deben facilitar la apropiación de los contenidos matemáticos como un resultado producido en la dinámica del proceso de enseñanza-aprendizaje de la Matemática General, mientras que las exigencias del futuro profesional están relacionadas con la necesidad de su desempeño en correspondencia con las demandas de una sociedad en constante desarrollo científico y tecnológico. Sin embargo, la repercusión de las insuficiencias reveladas en la enseñanza de los futuros profesionales, se manifiesta en las condiciones de vulnerabilidad económica, social y cultural las cuales afectan cuantitativamente los implicados en el proceso de formación, configuran un escenario que parece desafiar las metas propuestas en el plan de formación de cuadro desde el año 2012 hasta el 2020 (Angola, 2012).

Estos desafíos de pertinencia se han convertido ya en compromisos imprescindibles para superar las limitaciones epistemológicas, porque el proceso de formación matemática exige constante perfeccionamiento, lo cual requiere el desempeño eficiente de los futuros profesionales en un mundo en constante dinámica, que se desarrolla vertiginosamente en función de los Proyectos de Desarrollo de Ciencia y Tecnologías (PDCT), quienes la practican (Angola, 2016). En este sentido, en función de superar las limitaciones epistemológicas en la formación investigativa, se publicó como noticia de mayor transcendencia el desarrollo del Consejo Nacional de la Ciencia, Tecnología e Innovación (CNCTI), la Dirección Nacional de Desarrollo Tecnológico e Innovación (DNDTI); el Centro Tecnológico Nacional (CTN) en "la sociedad angoleña para contribuir al desarrollo del proceso de formación investigativa de los futuros profesionales" (Gómez, 2015, p. 1), con la implementación de políticas que combinan las investigaciones de los procesos económicos, con las transformaciones del sistema de dominación para contribuir al crecimiento social del país. Por esta razón, para hacer que, las tecnologías computacionales en la dinámica del proceso de formación matemática angoleña superen las limitaciones epistemológicas es imprescindible para el MESCTI elaborar un plan especial de superación profesional el cual tome en cuenta las expectativas, competencias y necesidades específicas de los profesionales y de respuesta a este encargo social de las IES, que puede dotar de ventajas para las empresas que no se sitúan en la hélice (empresa, MESCTI y las IES) mencionadas, pero cumplen más de una de sus funciones específicas, las cuales dan cuenta de una nueva realidad (Figura 1). 


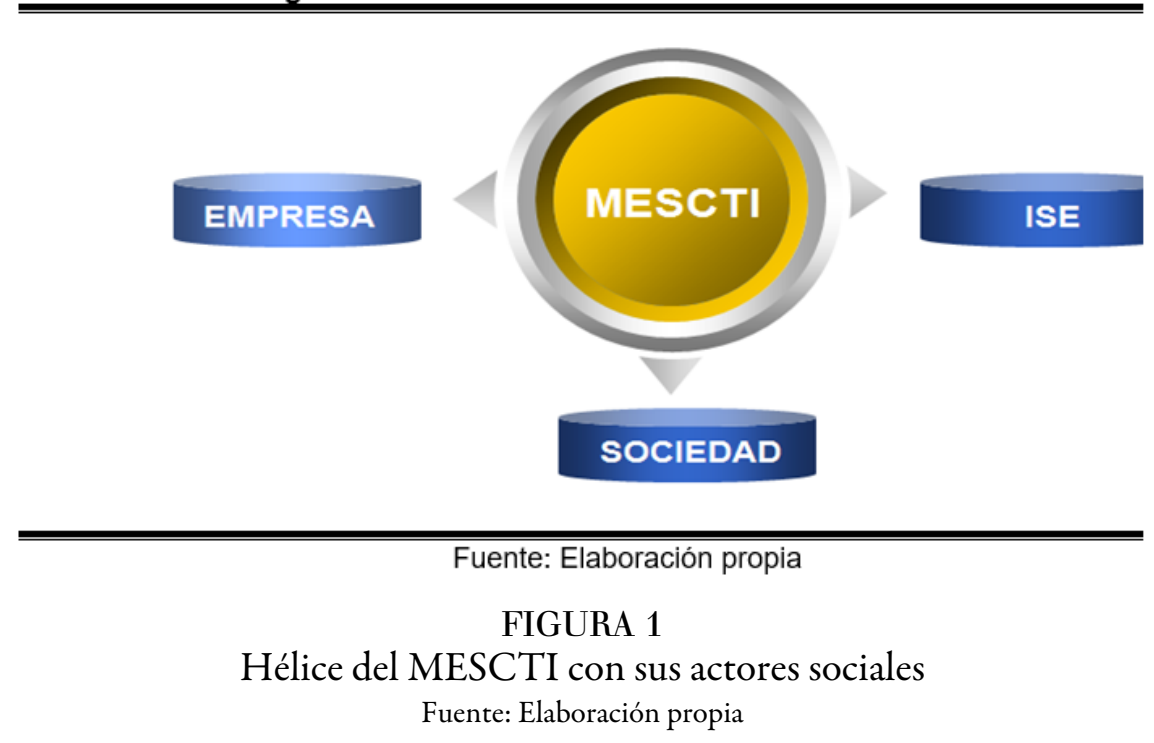

La relación de la dimensión en la sociedad angoleña estimula a nuevas políticas de diseñar incubadoras de empresas en las IES, a fin de sistematizar los conocimientos teóricos relacionados con los diversos procesos sustantivos vinculados con la búsqueda de la calidad y el logro de los resultados del proceso de enseñanzaaprendizaje para contribuir en la formación de un profesional mucho mejor preparado y comprometido con la sociedad.

Por otro lado, la creación de infraestructuras para el CTN, PCT, DNDTI, ENCTI y los PDCT, por el MESCTI, son considerados en la República de Angola, prioridades para estimular el desarrollo a alto nivel de las IES y marca una pauta en la sociedad del conocimiento y a su vez contribuirá al fortalecimiento de la formación investigativa, bajo un paradigma alternativo sobre función estratégica hacia un desarrollo con nuevas prioridades que enaltecerá los grandes desafíos de la soberanía angoleña. Esto, presupone un mayor empeño de los futuros profesionales en las tecnologías computaciones, al proceso de enseñanza-aprendizaje y traerá importantes transformaciones pertinentes en la dinámica del proceso de formación investigativa de calidad. No obstante, el colectivo de autores de la presente investigación fundamenta que, a partir de las insuficiencias en las IES, es necesario para el MESCTI implementar sin demoras, sistemas de verificación de evidencias del grado de cumplimiento, eficiencia, eficacia y efectividad de los procesos de gestión de autoridades para establecer oportunidades de mejoras a través de una formación matemática más significativa.

El concepto de aprendizaje significativo es desarrollado por David Paul Ausubel enmarcado en el paradigma cognitivo (Faustino, 2014, p. 47). Se asume en el presente artículo, como fundamento del trabajo referido al desarrollo del concepto de la enseñanza-aprendizaje y a la posición asumida por los futuros profesionales en la interpretación de los problemas en la formación matemática, la cual requiere la aplicación de recursos tecnológicos para una atribución significativa.

La formación matemática es un espacio de construcción de significados y sentidos entre los sujetos implicados en ella. Cuando este se desarrolla desde una pedagogía crítica, basada en los supuestos teóricos de Paulo Freire, donde la lógica que siguen los futuros profesionales para apropiarse y profundizar en la dinámica del proceso de enseñanza-aprendizaje de la matemática, no es la misma que ha tenido esta disciplina. Sin embargo, la autonomía de este proceso se obtiene cuando es favorecido por la secuencia dada por la lógica formal, por lo cual se "evidencia la necesidad de acercar la lógica formal de la matemática a la lógica de apropiación de nuevos contenidos”, siempre aplicando los avances de la ciencia y la técnica en su enseñanza (Faustino 2014, p. 56). 
Faustino, Pérez, Diéguez (2014), Faustino, Del Pozo y Arrocha (2014) demuestran que actualmente la formación matemática de los profesionales está sujeta a las demandas específicas de los avances de la ciencia y técnica, la cual tiende a privilegiar el desarrollo tecnológico con el progreso de la economía en las comunidades, a partir del incentivo de la formación del capital intelectual, vinculado a la producción de conocimientos. Dicha cuestión se considera fundamental para integrar la aplicación de los avances de la ciencia y técnica, en la dinámica del proceso de formación investigativa. Cuando la enseñanza-aprendizaje apunta únicamente al cálculo matemático algunos futuros profesionales obtienen buenos resultados en su evaluación; por otra parte fracasan cuando las situaciones presentadas son diferentes de las abordadas en su formación investigativa.

En este sentido, el juicio de Freire (1998) esclarece que "el investigador no es una cualidad o una forma de actuar" (p. 26) que se adiciona en la dinámica del proceso de enseñanza-aprendizaje. La práctica docente ofrece la indagación, la búsqueda, la investigación, la extensión y la internalización en su formación permanente. De esta forma, se asume en la presente investigación que el profesional docente investigador integra en su formación investigativa el desarrollo de individuos analíticos, críticos y reflexivos basada en el aprovechamiento del talento como estrategias de cambio social y de autoformación.

A su vez, autores como Faustino y Pérez, (2013) afirman que el análisis de las TIC, en el proceso de enseñanza-aprendizaje de la matemática, enmarca la pertinencia de las herramientas informáticas para viabilizar la apropiación de los contenidos los cuales pueden dinamizar el proceso de solución de problemas profesionales. En este caso para darle al contenido el carácter profesional, autores como Ramírez (2010) y Núñez (2012), también plantean un perfil importante para la formación que permite revelar la necesidad de transformar el medio social, mediante el reconocimiento de la pertinencia de la matemática en el proceso de solución de problemas profesionales aplicando las TIC, en la formación investigativa. La interrelación entre los componentes académico, investigativo y laboral en la formación investigativa es de carácter permanente, lo cual garantiza una coherencia en la articulación horizontal y vertical del proceso docente educativo en la carrera (Álvarez-Zayas, 1999). En efecto lo académico se desarrolla fundamentalmente mediante clases, donde predominan contenidos abstractos, modelados, simulados mediante el uso de las tecnologías computacionales. Lo laboral consiste en la práctica laboral, donde el contenido es la actividad de la producción y esta persigue integrar todos los contenidos, a través del empleo de métodos y tecnologías necesarias para desarrollar la actividad laboral de que se trate y la responsabilidad ante el trabajo. Así, lo investigativo se desarrolla a través del trabajo investigativo de los futuros profesionales, cuyo objetivo fundamental es contribuir a la adquisición de conocimientos y al desarrollo de habilidades para contribuir a la independencia cognoscitiva, la cual promueve su creatividad.

Al contrario, las tecnologías computacionales en el sector educativo angoleño, han dejado su identidad tradicional, como nuevo producto en la cultura, para ser utilizados por cualquier sector industrial viabilizando así, la promoción de bienes de servicios públicos y privados. Pero, la proliferación de formatos y plataformas de acceso a las tecnologías "favorecen el crecimiento de la información y contribuyen al desarrollo del proceso de enseñanza-aprendizaje en las instituciones" como lo plantea Núñez (2012, citados por Faustino, Pérez y Diéguez, 2013, p.97). Se requiere de la implementación de nuevos paradigmas educativos en la sociedad angoleña, lo cual enfatiza en las TIC, para el desarrollo de las capacidades intelectuales al cumplimiento del encargo social.

En realidad, este nuevo paradigma educativo en la sociedad angoleña, en función de contribuir en la eficiencia de la aplicación de métodos dinámicos y minimizar las limitaciones epistemológicas en el proceso de enseñanza-aprendizaje de la matemática, demanda más trabajo de los profesionales que una formación basadas en los procedimientos tradicionalistas. Además, los futuros profesionales adquieren nuevas experiencias y más habilidades intelectuales, pues son los profesionales quienes tienen la responsabilidad de diseñar actividades apropiadas, las cuales permiten potenciar las destrezas en su proceso de formación 
investigativa. Esto lleva a tomar la decisión de cómo y cuándo se pueden usar de manera adecuada las tecnologías computacionales en la formación matemática para minimizar las insuficiencias epistemológicas.

Es preciso considerar el papel de las tecnologías computacionales y la sistematización de métodos dinámicos, porque constituyen herramientas mediadoras del proceso de enseñanza-aprendizaje en la formación profesional. Sin embargo, en la educación angoleña, al abordar la dinámica de este proceso, no se hace suficiente énfasis en las tecnologías computacionales para dinamizar los procesos del pensamiento, requeridos para dar solución a los problemas reales de la profesión que demandan una lógica en consonancia con la búsqueda de la información matemática propiciadora, del necesario vínculo entre el análisis del problema profesional y el contexto laboral. Lo cual implica reorientar el tratamiento de los objetivos, contenidos y métodos con las tecnologías computacionales en la dinámica del proceso de enseñanzaaprendizaje de la matemática, a tono con el desarrollo científico-técnico.

La propuesta de Quitembo (2010), acerca de una nueva IES más pertinente y mejor orientada al dar respuesta a necesidades sociales, cobra en este contexto especial vigencia. Es decir, una formación investigativa que es juzgada en términos de su contribución en el desempeño de la economía nacional y a través de ellos al mejoramiento de las condiciones de vida. De ahí que, es imprescindible integrar la ciencia y la técnica, vincularla con la apropiación de una metodología coherente para la solución de los problemas profesionales; cuestión reconocida por los autores: Faustino, Wongo y Craib (2015b) lo cual puede dificultar el desarrollo de las habilidades de los futuros profesionales en las aplicaciones de los conocimientos técnico-científicos y en su desempeño profesional. Faustino, Pérez y Diéguez (2013), confirman que:

(...) es necesario llevar a los futuros profesionales en la sistematización de los conocimientos técnico-científicos, porque requieren un proceso de contextualización de los contenidos y los fenómenos que ocurren en la sociedad, teniendo en cuenta las diferencias individuales e institucionales, enriquecidas en cualquier contexto por el desarrollo de un pensamientoinvestigativo directamente influenciado por los avances técnico-científicos. (p. 8).

De esta manera, es imprescindible estimular el uso de las tecnologías computacionales, como herramientas didácticas que permiten mejorar cuali-cuantitativamente el proceso de enseñanza-aprendizaje de la matemática, porque en la práctica, los futuros profesionales solo repiten lo que transmite el profesorado (profesionales). Es decir, someter el proceso de enseñanza-aprendizaje de técnicas sobre cálculos sin conocer su sentido y significado. Cuestión que necesita herramientas investigativas en el mejoramiento del desempeño eficiente del profesional en la solución de los problemas, mediante la integración de conocimientos y habilidades para el manejo de las TIC, en un proceso de búsqueda científica de los contenidos matemáticos.

Investigadores como Faustino y Wongo (2014) señalan que, el contenido en su versión digital en diferentes plataformas en los entornos educativos, posibilitan dinamizar el proceso de enseñanza-aprendizaje de la matemática. Aun así, las TIC, como mediadores didácticos en las IES públicas y privadas, en la sociedad angoleña no han sido suficientemente potenciadas por metodologías del proceso investigativo, como elemento fundamental en la búsqueda de los contenidos matemáticos en su versión digital, por falta de dominio de las tecnologías computacionales que frena el desarrollo del pensamiento matemático investigativo del alumnado.

En este sentido, es necesario considerar los contenidos matemáticos en su versión digital como mediadores didácticos, porque pueden contribuir a la transformación del sujeto en un individuo más independiente, sobre todo si están confeccionados con los recursos informáticos de avanzada y orientan a los futuros profesionales hacia un rasgo esencial del objeto de su investigación. De esta manera, los mediadores didácticos confeccionados por las TIC, también contribuyen al proceso de identificación de los conceptos matemáticos por aprender.

Es preciso vincular los recursos informáticos con la llamada tecnología de aprender a pensar de acuerdo con los fundamentos de Fuentes (2009), lo cual afirma que permiten al futuro profesional viabilizar su proceso de descubrimiento de principios y reglas lógicas, las cuales se mueven desde la inferencia, deducción hasta la creación del pensamiento, mediante el tránsito necesario que plantea Vigotsky (1989), en su teoría del 
conocimiento. Se señala como elemento importante, la necesidad de relacionar el contenido, con los llamados procesos de internalización del pensamiento lógico, cuestión fundamental para integrar las tecnologías computacionales y los métodos activos en la dinámica del proceso de enseñanza-aprendizaje de la matemática, que contribuyen al desarrollo del pensamiento-investigativo, lo cual demanda las actuales exigencias del contexto adecuado a la realidad individual.

Se considera un aspecto clave para minimizar las limitaciones epistemológicas antes referenciadas: la necesidad de reestructurar los objetivos del plan nacional de formación de cuadros (2013-2020), constatadas en la Ley de Bases del Sistema de Educación, 2001, que se utilizan en las IES, para enfrentar la actual crisis económica provocada por el bajo rendimiento económico del petróleo en el mercado mundial, la cual también dificulta la compra de tecnologías de avanzada para perfeccionar el desempeño del profesional en sus investigaciones en la sociedad angoleña.

Sobre los retos de la dinámica del proceso de enseñanza-aprendizaje de la matemática, sustentado en las tecnologías computacionales, también han investigado varios autores como Pardo (2004), Papanastasiou y Ferdig (2006), Cruz-Pichardo y Puentes-Puente (2012), Faustino y Pérez. (2013), Gungula, DieguezBatista y Ugartemendía (2015), quienes demuestran la pertinencia de la utilización metodológica de las tecnologías computacionales en la formación matemática, la cual puede contribuir a alcanzar niveles de calidad y efectividad de manera que proporcione un resultado científico, que satisfaga las exigencias actuales planteadas por la sociedad.

Sin embargo, a pesar de la importancia de las tecnologías computacionales en el estudiantado de la carrera de Licenciatura en Educación Matemática, aún es posible identificar un conjunto de insuficiencias que atentan contra la calidad del universitario angoleño, relacionadas con la aplicación de los métodos activos y asistentes matemáticos como: Mathcad professional, WinQSB, Maple18, Algebrator, MatlabR2010a, Georgebra, Python vs Math, Wolfram Mathematica8, Derive6 entre otros, de forma integrada, tal que garanticen la sistematicidad del contenido a desarrollar en el diagnóstico de los problemas profesionales vinculados con su especialidad, en un contexto determinado.

Por otro lado, en la práctica este proceso también tiene sus dificultades, por las limitaciones metodológicas, la carencia de superación tecnológica y el hecho de necesitar ordenadores para el cumplimiento de los objetivos preconizados por el MESCTI en la sociedad angoleña y fortalecer las políticas de cooperación con la IES a nivel nacional e internacional. Por ende, es imprescindible promover la superación constante de profesionales de la matemática en la utilización de las TIC, porque desempeña un papel vital para el manejo eficiente del contenido matemático indispensable para la práctica, pues constituyen sustentos cognitivos en las interpretaciones del proceso de solución de problemas profesionales.

En esta evaluación se adjudica valorar a las TIC, como medio didáctico el cual facilita minimizar las insuficiencias en la dinámica del proceso de enseñanza-aprendizaje y contribuye al desarrollo de la cultura matemática, pues es necesario que los profesionales empleen las tecnologías computacionales, porque ellas refuerzan la comprensión de los problemas y promueven el interés por parte de sus estudiantes, a partir de la combinación de métodos de enseñanza-aprendizaje logrando la profundización del contenido y un mejor autoaprendizaje en su proceso formativo.

Lo anterior exige procedimientos y técnicas para lograr una adecuada proyección metodológica del uso de los mediadores didácticos, como herramientas informáticas las cuales facilitan la orientación de los conocimientos matemáticos. Es necesario asumir en el presente artículo los fundamentos de Faustino (2014), donde revela la significación de la base de la teoría del conocimiento científico y la sistematización de las tecnologías computacionales, como elemento esencial en la enseñanza superior.

Los mediadores didácticos confeccionados por las tecnologías computacionales en la enseñanza superior son pertinentes porque contribuyen a la transformación del futuro profesional en un individuo más independiente y orientan hacia un rasgo esencial en la formación de conceptos matemáticos a través de la visualización de gráficos e imágenes de funciones tridimensionales de calidad y de alto grado de interactividad, 
mostrados por las TIC, que permite aclarar el pensamiento-investigativo ${ }^{[1]}$ y fortalece lo imaginológico en el proceso de enseñanza-aprendizaje de la matemática, como un elemento dinamizador en la innovación.

Las políticas de integración de las TIC en el proceso de enseñanza-aprendizaje de la matemática como mediadores didácticos, en las IES, son de vital importancia en la apreciación de rasgos esenciales para resolución de problemas, favoreciendo un aprendizaje desarrollador, con la aplicación de métodos activos en el cual el futuro profesional no solo gráfica las funciones observadas sino, identifica conceptos equivocados como un proceso de innovación en la formación matemática. Las tecnologías computacionales en la dinámica del proceso de formación matemática, también facilitan la apropiación constructiva de los conocimientos teórico-prácticos y el contenido, los cuales a su vez permitirán los procesos de autoevaluación en su formación investigativa y se reflexionará sobre el estado de ese aprendizaje.

Las políticas de integración de las TIC en la formación matemática, como elemento visualizador en la dinámica del proceso de enseñanza-aprendizaje de la matemática, tienen importancia para la formación del pensamiento-investigativo y el desarrollo de su independencia cognoscitiva (Figura 2). Corroborando lo antes afirmado, se asume una conclusión dada por Frómeta-Quintana (2007), en cuya investigación se establece que la metodología interpretativa visual, sustentada en la concepción icónica hermenéutica, se erige como un instrumental válido por su lógica interna y el nivel de organicidad, los que permiten la dirección científica del proceso de mediación semiótica visual mediante el cual futuros profesionales construyen la cultura y transforman su mundo a partir de su relación con la imagen referida que rescatan los estadios de creación del conocimiento y sus características de desarrollo.

Figura 2: Las políticas de integración de las TIC en la formación matemática

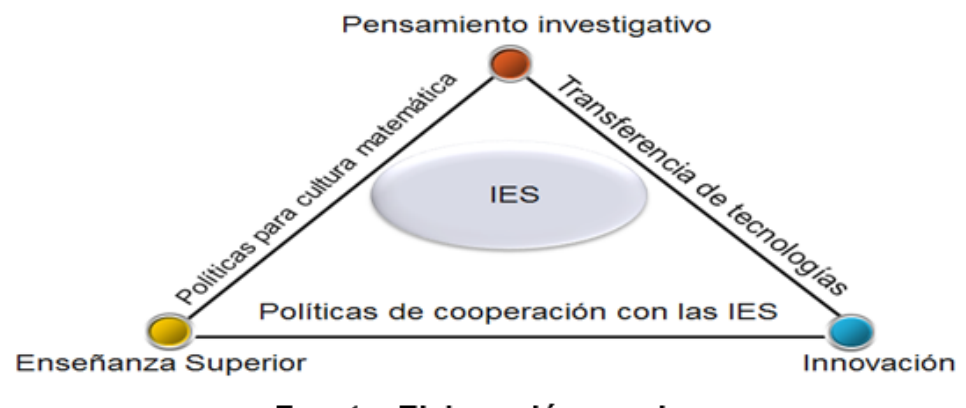

Fuente: Elaboración propia

FIGURA 2

Las políticas de integración de las TIC en la formación matemática Fuente: Elaboración propia

En la esencia dialéctica; los mediadores didácticos confeccionados por las tecnologías computacionales, pueden evidenciar lo dinámico en las investigaciones, al estar en constante transformación debido al ritmo y crecimiento del desarrollo social. La creación de herramientas tecnológicas para la formación puede facilitar la transferencia tecnológica. Pues la calidad que exige las IES requiere cambios de época, para lograr hacerse visible en las universidades pioneras las cuales están tomando correctivos necesarios y adaptando sus procesos de gestión en su formación investigativa (Tünnermann, 2011).

En esta formación investigativa están involucrados profesionales y futuros graduados, los cuales constituyen elementos móviles en la dinámica del proceso de enseñanza-aprendizaje, pero otros factores han de tenerse en cuenta para lograr que cada uno de los elementos antes mencionados, logre sus objetivos en su formación y contribuyan a viabilizar la sistematización del contenido matemático. No se trata de sobrevalorar las potencialidades de las TIC en la formación profesional, sino de aprovechar al máximo sus beneficios y no subvalorar en su totalidad los medios tradicionales en la formación matemática. 


\section{Las Tecnologías de Información y Comunicación en la dinámica del proceso de enseñanza-aprendizaje de la matemática en las IES.}

La incursión de las herramientas tecnológicas para los futuros profesionales en el proceso de enseñanzaaprendizaje de la matemática angoleña, actualmente impone una dinámica de transformación socio-cultural concerniente a la praxis pedagógica y didáctica de la matemática. Esto presupone una cultura pedagógica y didáctica basada en las TIC para dinamizar el proceso, pero también de la sistematización de posturas teórico-prácticas contextualizadas en la profesión, sustentadas en procedimientos que permitan la formación de una cultura tecnológica investigativa. Entendida en el presente artículo como una actividad orientada a la generación de nuevos conocimientos tecnológicos, que pueden ser aplicados directamente a la producción, distribución de servicios y posibilita el proceso de orientación a investigaciones de avanzada.

Entender la cultura tecnológica en las investigaciones de avanzada en que se mueve el mundo actual, implica no solo el reconocimiento de los avances de las TIC, sino también, su articulación en la dinámica del proceso de enseñanza-aprendizaje de la matemática, vinculados a los procesos de la producción en el sector social; porque las TIC, proporcionan múltiples formas de representar problemáticas en lo cotidiano que permiten a los futuros profesionales desarrollar estrategias de resolución de problemas y posibilita la comprensión de los conceptos matemáticos, lo cual están trabajando (Faustino, Wongo y Craib 2015b).

A raíz de estos cambios; la dinámica del proceso de formación matemática enfrenta un nuevo paradigma de enseñanza-aprendizaje, que implica la utilización e innovación de metodologías para la formación de conceptos matemáticos mediadas por las tecnologías computacionales y la incorporación de nuevos roles docentes con mayor responsabilidad, flexibilidad y colaboración en la construcción del conocimiento lo cual viabiliza la autonomía de los futuros profesionales en su proceso de investigación, sin embargo, a pesar de la evidente introducción de las TIC en las IES, los futuros graduados angoleños aún presentan limitaciones, que no les permiten la generalización de los conocimientos en nuevas situaciones para solucionar problemas con el uso de las computadoras, internet y asistentes matemáticos.

El uso de las herramientas tecnológicas en la enseñanza-aprendizaje tiene como propósito innovar ${ }^{[2]}$ y mejorar la calidad del proceso, de tal forma que los profesionales en los niveles superiores de enseñanza no deben desestimar las transformaciones ocurridas con la implementación adecuadas de las TIC. Para un uso eficiente en la dinámica del proceso de enseñanza-aprendizaje de la matemática, se requiere una preparación tecnológica de forma sistemática en los profesionales, cuestión valorada también por (Quesada, 2010).

En este sentido el siguiente juicio de Faustino (2014) afirma que, a pesar de incorporar la "disponibilidad de los recursos tecnológicos” (p. 33) la práctica docente en el aula no ha variado mucho los procedimientos tradicionalistas, en el uso de las tecnologías computacionales, para transmitir la información, por las limitaciones epistemológicas en la sistematización de métodos activos y las tecnologías al proceso de solución de problemas. Por consiguiente, los procesos de innovación en la dinámica del proceso de enseñanzaaprendizaje de la matemática, constituyen motivos de preocupación para el cumplimiento de los objetivos propuestos por la educación superior angoleña en diseñar estrategias que el futuro profesional sea capaz de crear situaciones, las cuales favorezcan el aprendizaje significativo con el apoyo de asistentes matemáticos en su formación.

El uso adecuado de las tecnologías computacionales, donde se encuentran asistentes matemáticos como Mathcad professional, WinQSB, Maple18, Algebrator, MatlabR2010a, Georgebra, Python vs Math, Wolfram Mathematica8, Derive6 y los modelos estadísticos, en la dinámica del proceso de enseñanzaaprendizaje de la matemática, facilitan a los futuros profesionales a realizar acciones significativas para la modelación de problemas matemáticos los cuales contribuyen a solucionar las necesidades sociales. En el presente artículo, cuando se habla de asistentes matemáticos se está haciendo referencia a una concepción de herramientas informáticas aplicadas en la dinámica del proceso de enseñanza-aprendizaje de la referida 
Ciencia, las cuales deben centrar el desarrollo de software con sistema de conocimientos y acciones que dinamizan todo el proceso, en instituciones afines a la profesión de los angoleños.

Pero, para que el futuro profesional realice dichas acciones, en la dinámica del proceso de enseñanzaaprendizaje de la matemática, es necesario incentivar a los profesionales la integración de las TIC y los medios tradicionales para la manipulación eficiente de los fenómenos que ocurren en el contexto educativo, permitiendo una visión más amplia y profunda del contenido matemático cuando son bien gestionados en la dinámica del proceso.

El colectivo de investigadores de la presente pesquisa fundamenta que en este proceso de interacción entre los asistentes y el objeto de conocimiento teórico matemático se puede concebir, la enseñanza-aprendizaje, como significativa porque es un proceso de adquisición de sentidos y significados a través del establecimiento de relaciones sustantivas entre los conocimientos previos que son necesarios considerar antes de solucionar el problema matemático planteado y explicar de forma clara a los alumnos-alumnas sobre la significación práctica de la situación apoyados en las herramientas informáticas.

En este sentido, se sugiere seleccionar y estructurar el contenido teórico matemático de modo que propicie la comprensión de los problemas planteados, no solo teniendo en cuenta el punto de vista de las asignaturas en la dinámica del proceso de enseñanza-aprendizaje, sino considerando el nivel de dificultad que ofrecen los futuros profesionales en el uso de las tecnologías para la solución de problemas lo cual implica la integración de herramientas informáticas, logrando mayor pertinencia en la dinámica del proceso de enseñanza-aprendizaje de la matemática.

Esta dinámica del proceso presenta insuficiencias en la utilización de las tecnologías, cuestión que se particulariza en los métodos inadecuados para la aplicación de las TIC y en la solución de problemas profesionales. Se requiere, entonces desarrollar en los futuros profesionales, las habilidades de aplicar las TIC de forma independiente y creadora en la solución de situaciones problemáticas relacionadas con el objeto de trabajo en la profesión, como expresión del desarrollo de las capacidades profesionales en la solución de tales problemas.

Los futuros profesionales desarrollan la habilidad de aplicar las TIC en la dinámica del proceso de enseñanza-aprendizaje de la matemática y en la solución de problemas profesionales. Es imprescindible la responsabilidad de los profesionales en la utilización de métodos que posibilitan el desarrollo de una dinámica caracterizada por la necesidad de formar universitarios, capaces de analizar problemáticas planteadas y buscar la información necesaria para precisar las vías de solución con la utilización de las TIC en la interpretación de los resultados obtenidos en el campo de acción profesional en los cuales fueron planteados.

\section{Utilización de las tecnologías computacionales en la formación matemática en las IES.}

Los estándares más desarrollados permiten compatibilizar el contenido matemático en diferentes plataformas y entornos educativos, lo que posibilita mejorar cualitativamente el proceso de enseñanzaaprendizaje de la matemática, sin embargo, durante el movimiento del proceso, sustentado en las tecnologías computacionales o TIC, los mediadores didácticos no han sido suficientemente potenciados, por lo cual se manifiestan insuficiencias en el desarrollo del pensamiento investigativo, como elemento fundamental en la apropiación singular de los contenidos matemáticos con la utilización de las TIC.

La responsabilidad de los profesionales, ante las políticas de mejoramiento que intervienen en la didáctica de la matemática actualmente, deben establecer conciencia en la comunidad educativa, sobre el conocimiento e incidencia en la aplicación de las TIC. Para lo $\operatorname{corar}$ una reflexión acerca de la necesidad de desarrollar una cultura tecnológica en los futuros profesionales, que les permita desde la sistematización de los conocimientos y métodos de la investigación científica solucionar los problemas profesionales con independencia en la dinámica del proceso de enseñanza-aprendizaje de la matemática aplicando las TIC. 
Asimismo el encargo de los profesionales en la formación matemática, reviste una importancia significativa, como lo planteó Santos (1997) y Diéguez (2001) al decir que en la dinámica del proceso de enseñanza-aprendizaje de la matemática, durante la experiencia de los futuros profesionales en la solución de problemas, es fundamental desarrollar una disposición la cual, les permita participar en actividades propias del quehacer matemático investigativo de forma dinámica aplicando las tecnologías. En este contexto, es necesario que los universitarios aprendan a resolver problemas aprovechando las potencialidades de las tecnologías computacionales en diversas situaciones de la vida desde diferentes ángulos para comprender el contenido matemático.

El conocimiento, en el caso particular de la Matemática General, se caracteriza por ser abstracto y secuencial. Se trata de una disciplina acumulativa, unas actividades exigen otras previas, lo cual demanda comprensión lógica y memoria comprensiva de los contenidos anteriores. Esto es, saber aplicar los conceptos y los procedimientos en acción, tener desarrollado el pensamiento investigativo de acuerdo con sus exigencias, por lo cual se hace necesaria una concientización por parte de profesionales y futuros profesionales, la utilización de las tecnologías computacionales y sus requerimientos, para lograr la apropiación del contenido matemático.

La reflexión y práctica en la responsabilidad de los profesionales impulsan a los futuros profesionales en su quehacer ma $\neg$ temático investigativo de forma dinámica, cons-tituye en gran parte del cambio esencial en in $\neg$ centivar las IES angoleñas para la elaboración de planes de formación, los cuales integran herramientas informáticas con problemas contextualizados, que respalden ambientes de aprendizaje pertinentes en la dinámica del proceso de enseñanza-aprendizaje de la matemática en todas las carreras donde se imparten dichos contenidos (Rico y Gil, 2003) cuestión valorada también por (Calero, 2008).

Lo anteriormente valorado, requiere la integración de los procesos de innovación en la dinámica del proceso de enseñanza-aprendizaje de la matemática, para lograr no solo mayor pertinencia con el uso de las tecnologías, sino de desarrollar asistentes matemáticos con problemas contextualizados a su realidad.

Para alcanzar esta meta, debe producirse un cambio en la concepción tradicional de la dinámica del proceso de enseñanza-aprendizaje de la matemática en y una nueva comprensión acerca de cómo las tecnologías computacionales, pueden ayudar a crear nuevos entornos de aprendizaje en que los futuros profesionales se sientan comprometidos y asuman mayores roles de responsabilidades sobre su propia formación con mayor independencia en la sistematización de los conocimiento matemáticos, siempre en interacción con las TIC.

La interactividad es un elemento destacable en el proceso de enseñanza-aprendizaje, utilizando las TIC, pues permite a los futuros profesionales ejercer una relación directa con los contenidos que está trabajando y manipularlos con mayor independencia, creando trabajos propios. Por otro lado, los profesionales pueden beneficiarse de esta interactividad en sus explicaciones utilizando software, como el Geogebra en la pizarra digital, cuestión valorada también por (Kozma, 2008).

Entonces, se requiere además de encontrar alternativas coherentes desde posturas pedagógicas con fundamentos en la didáctica de la matemática, tal que revelen pasos con rigor científico de cómo buscar la integración de las TIC, en los escenarios de aprendizaje para minimizar las limitaciones epistemológicas de los futuros profesionales universitarios angoleños, desde la historicidad de su formación matemática que no esté exenta de los problemas. Los cuales ocurren en la sociedad angoleña bajo los estilos de aprendizaje con procedimientos tradicionalistas y asistentes matemáticos con problemas descontextualizados de la realidad que no son portadores de actividades matemáticas investigativas.

De ahí que se reconoce, la responsabilidad de los profesionales en la dinámica del proceso de enseñanzaaprendizaje de la matemática, en buscar alternativas metodológicas al respecto y de medidas que contribuyan a mitigar las profundas insuficiencias epistemológicas existentes en la utilización de asistentes matemáticos con problemas descontextualizados, lo cual dificulta la solución de problemas reales (Tabla 1). Las referidas deficiencias epistemológicas, manifestadas al abordar esta dinámica, permiten afirmar que actualmente existen insuficientes asistentes con problemas contextualizados acerca de la apropiación de un contenido 
matemático portador de una lógica indagativa y métodos para solución de problemas dados en el contexto profesional concreto.

TABLA 1

Porcentaje de la utilización de asistentes matemáticos con problemas descontextualizado

Tabla 1: Porcentaje de la utilización de asistentes matemáticos con problemas descontextualizados

Compromiso de los profesionales en la utilización de las TIC y medios tradicionales en el proceso de formación matemática

\begin{tabular}{|c|c|c|c|c|c|c|c|c|c|c|c|c|}
\hline \multicolumn{6}{|c|}{ profesionales } & \multicolumn{7}{|c|}{ futuros profesionales } \\
\hline \multicolumn{3}{|c|}{$\begin{array}{c}\text { Reglas de cálculo, } \\
\text { pizarrón y tablas } \\
\text { matemáticas }\end{array}$} & \multicolumn{3}{|c|}{$\begin{array}{c}\text { Asistentes } \\
\text { matemáticos } \\
\text { descontextualizados }\end{array}$} & \multicolumn{3}{|c|}{$\begin{array}{c}\text { Libretas, Folletos y } \\
\text { medios para la } \\
\text { escritura }\end{array}$} & \multicolumn{4}{|c|}{$\begin{array}{c}\text { Asistentes matemáticos } \\
\text { descontextualizados }\end{array}$} \\
\hline $\mathrm{Si}$ & No & Total & $\mathrm{Si}$ & No & Total & $\mathrm{Si}$ & No & Total & Si & No & Total & Años \\
\hline $71 \%$ & $61 \%$ & $100 \%$ & $23 \%$ & $77 \%$ & $100 \%$ & $91 \%$ & $9 \%$ & $100 \%$ & $34 \%$ & $66 \%$ & $100 \%$ & 2013 \\
\hline $79 \%$ & $21 \%$ & $100 \%$ & $26 \%$ & $74 \%$ & $100 \%$ & $94 \%$ & $6 \%$ & $100 \%$ & $21 \%$ & $79 \%$ & $100 \%$ & 2014 \\
\hline $83 \%$ & $17 \%$ & $100 \%$ & $31 \%$ & $69 \%$ & $100 \%$ & $95 \%$ & $5 \%$ & $100 \%$ & $16 \%$ & $84 \%$ & $100 \%$ & 2015 \\
\hline $89 \%$ & $11 \%$ & $100 \%$ & $42 \%$ & $58 \%$ & $100 \%$ & $90 \%$ & $\begin{array}{l}10 \\
\%\end{array}$ & $100 \%$ & $10 \%$ & $90 \%$ & $100 \%$ & 2016 \\
\hline $91 \%$ & $9 \%$ & $100 \%$ & $15 \%$ & $85 \%$ & $100 \%$ & $98 \%$ & $2 \%$ & $100 \%$ & $8 \%$ & $92 \%$ & $100 \%$ & 2017 \\
\hline $91 \%$ & $9 \%$ & $100 \%$ & $15 \%$ & $90 \%$ & $100 \%$ & $98 \%$ & $2 \%$ & $100 \%$ & $6 \%$ & $94 \%$ & $100 \%$ & 2018 \\
\hline
\end{tabular}

Fuente: Elaboración propia del colectivo de autores (2018).

Fuente: Elaboración propia del colectivo de autores (2018).

No obstante, la carencia de asistentes matemáticos con problemas contextualizados acerca de la aplicación de cuestionarios validados con anterioridad en el diagnóstico inicial en las IES reveló que, actualmente limitan la efectividad del desarrollo de la dinámica del proceso de enseñanza-aprendizaje de los alumnosalumnas en la carrera de Licenciatura en Educación Matemática (Tabla 2). Por consiguiente, resulta necesaria la estimulación de crear tecnologías centradas en los problemas contextualizados en la profesión, lo cual reviste una característica muy particular que se expresa en la necesidad de un espacio en la formación matemática para el despliegue de dicha contextualización. 
TABLA 2:

Porcentaje de la carencia de asistentes matemáticos contextualizados

Tabla 2: Porcentaje de la carencia de asistentes matemáticos contextualizados

\begin{tabular}{|c|c|c|c|c|c|c|c|c|c|c|c|c|}
\hline \multicolumn{6}{|c|}{ profesionales } & \multicolumn{7}{|c|}{ futuros profesionales } \\
\hline \multicolumn{3}{|c|}{$\begin{array}{c}\text { Asistentes } \\
\text { matemáticos } \\
\text { descontextualizados }\end{array}$} & \multicolumn{3}{|c|}{$\begin{array}{c}\text { Asistentes } \\
\text { matemáticos } \\
\text { contextualizados }\end{array}$} & \multicolumn{3}{|c|}{$\begin{array}{c}\text { Asistentes } \\
\text { matemáticos con } \\
\text { problemas } \\
\text { descontextualizados }\end{array}$} & \multicolumn{4}{|c|}{$\begin{array}{l}\text { Asistentes matemáticos con } \\
\text { problemas contextualizados }\end{array}$} \\
\hline Si & No & Total & $\mathrm{Si}$ & No & Total & $\mathrm{Si}$ & No & Total & Si & No & Total & Años \\
\hline $38 \%$ & $62 \%$ & $100 \%$ & $\%$ & $84 \%$ & $100 \%$ & $50 \%$ & $50 \%$ & $100 \%$ & $42 \%$ & $58 \%$ & $100 \%$ & 2013 \\
\hline $28 \%$ & $72 \%$ & $100 \%$ & $23 \%$ & $77 \%$ & $100 \%$ & $53 \%$ & $47 \%$ & $100 \%$ & $66 \%$ & $34 \%$ & $100 \%$ & 2014 \\
\hline $19 \%$ & $81 \%$ & $100 \%$ & $26 \%$ & $74 \%$ & $100 \%$ & $45 \%$ & $55 \%$ & $100 \%$ & $76 \%$ & $24 \%$ & $100 \%$ & 2015 \\
\hline $16 \%$ & $84 \%$ & $100 \%$ & $31 \%$ & $69 \%$ & $100 \%$ & $83 \%$ & $17 \%$ & $100 \%$ & $84 \%$ & $16 \%$ & $100 \%$ & 2016 \\
\hline $12 \%$ & $88 \%$ & $100 \%$ & $42 \%$ & $58 \%$ & $100 \%$ & $90 \%$ & $10 \%$ & $100 \%$ & $90 \%$ & $10 \%$ & $100 \%$ & 2017 \\
\hline $9 \%$ & $91 \%$ & $100 \%$ & $56 \%$ & $44 \%$ & $100 \%$ & $91 \%$ & $9 \%$ & $100 \%$ & $92 \%$ & $8 \%$ & $100 \%$ & 2018 \\
\hline
\end{tabular}

Fuente: Elaboración propia del colectivo de autores (2018).

Fuente: Elaboración propia del colectivo de autores (2018).

En este sentido, se reconoce claramente que la dinámica del proceso de enseñanza-aprendizaje, según el diagnóstico aplicado carece de aspectos estructurales y normativos, los cuales posibilitan desarrollar herramientas informáticas de manera más adecuada donde el futuro profesional sea capaz de actuar consciente y creadoramente en la realización de sus tareas. Por tanto, para lograr ese propósito es imprescindible garantizar su preparación como sujeto activo desde su propia formación para transformar la realidad en que vive.

Por otra parte, también se realizó el diagnóstico mediante encuestas validadas con anterioridad a una población participante de graduados desde el año 2015 hasta el 2018 en segunda versión y entidades laborales de los mismos, bien como valoraciones efectuadas con el MESCTI y actualmente frente las agudas deficiencias que se manifiestan en el país se realizó un estudio para identificar la escasez de cuadros; cuyos resultados indican que la carencia de profesionales en el área de las tecnologías y las insuficiencias en los aspectos estructurales, imposibilitan el desarrollo de herramientas informáticas para la dinámica del proceso de enseñanza-aprendizaje de la matemática para estimular el desarrollo del pensamiento (Faustino, PérezSánchez y Gungula, 2017).

A pesar de estas concepciones, se observan carencias de profesionales calificados, debido a las insuficiencias de cursos de maestría y doctorados en las IES vinculados con las TIC (Tabla 3). De ahí que la intencionalidad fundamental en la dinámica del proceso de enseñanza-aprendizaje de la matemática sea, la motivación del espíritu creador, investigativo, estrechamente ligado al desarrollo de las tecnologías, en conjunto con los problemas contextualizados en la producción. Ellos presuponen un proceso de formación participativa, donde el futuro profesional sea el centro del proceso y sujeto activo en el mismo. Por tanto, el empleo de las TIC en la formación matemática, es de vital importancia, razón por la cual la formación tiene que dejar de ser informadora y convertirse en desarrolladora tal que resulte de una práctica cotidiana orientada con el propósito de minimizar las necesidades del profesional calificado. 
TABLA 3

necesidades del profesional cualificado

Tabla 3: necesidades del profesional cualificado

\begin{tabular}{|c|c|c|c|c|}
\hline \multirow[t]{3}{*}{ Necesidades } & \multicolumn{4}{|l|}{ Años académicos } \\
\hline & 201 & 201 & 201 & 201 \\
\hline & 5 & 6 & 7 & 8 \\
\hline Escasez de profesionales calificado & $78 \%$ & $89 \%$ & $90 \%$ & $95 \%$ \\
\hline Necesidad del profesor calificado & $90 \%$ & $95 \%$ & $95 \%$ & $89 \%$ \\
\hline $\begin{array}{l}\text { Oferta de servicios del profesor } \\
\text { calificado }\end{array}$ & $94 \%$ & $95 \%$ & $95 \%$ & $91 \%$ \\
\hline
\end{tabular}

Fuente: MESCTI (Febrero, 2015).

Fuente: MESCTI (Febrero, 2015).

La vigente necesidad de formar un profesional calificado en la sociedad angoleña, el MESCTI, volvió a revisar los aspectos normativos, con el objetivo de establecer un proceso de superación de los profesionales existentes y la restructuración de los nuevos planes de estudio para garantizar aún más la formación de especialistas de perfil amplio, con la implementación de las TIC, en las carreras universitarias como vía de viabilizar una formación integral más sólida de los futuros profesionales. Sin embargo, en la práctica, no se ha logrado superar las deficiencias en el dominio de las TIC para dinamizar la utilización de métodos en la enseñanza-aprendizaje de la matemática.

Aparte de las graves deficiencias normativas, en la formación matemática angoleña es necesario reconocer también la escasez de las investigaciones centradas en el diseño, gestión y desarrollo de herramientas informáticas para la dinámica del proceso de enseñanza-aprendizaje de la matemática, que cubran los diversos contenidos matemático-didácticos requeridos actualmente, bien como la necesidad de superar los profesionales en conocimientos matemáticos y tecnológicos como planteó (Fullan, 2001).

Para minimizar la falta de profesionales calificados en las IES angoleñas, se crean políticas para la estimulación de la formación y superación profesional con grados de maestrías y doctorados e insertarse en los avances científico-tecnológicos, pues hasta al momento la mayoría de los profesionales que imparten clases son extranjeros y tienden a suspender la docencia por la actual crisis económica provocada por la baja del petróleo en el mercado mundial.

De allí que es necesario promover eficazmente la formación investigativa y la autonomía en las IES en todas las carreras donde se imparten dichos contenidos, para que los futuros profesionales desarrollen el pensamiento investigativo y les permita de forma estratégica sistematizar los conocimientos precedentes en el proceso de solución de problemas, así como comprender el papel que desempeñan las matemáticas en el contexto económico, cultural, social y profesional.

Entonces, se necesita realizar profundas transformaciones en el perfeccionamiento del proceso de enseñanza-aprendizaje de la matemática, desde los aportes realizados en el campo de la pedagogía y la didáctica de la matemática, al mejoramiento del desempeño de los futuros profesionales en su proceso de investigación, desde todas las asignaturas de la carrera de forma sistematizada tal que, conlleve al reconocimiento de las potencialidades de las tecnologías computacionales en la formación.

Desempeño matemático con la aplicación eficaz de las tecnologías computacionales. El proceso de desempeño eficaz en la enseñanza-aprendizaje de la matemática, se puede lograr desde lo indagativo, mediante 
la realización de tareas, cuyo objetivo fundamental es contribuir a la adquisición de conocimientos teóricos para el desarrollo de habilidades en la solución de problemas, que contribuye fundamentalmente a la independencia cognoscitiva y al fomento de intereses en los universitarios con la apropiación de las TIC y dirigida al desarrollo de su creatividad a fin de minimizar las problemáticas existentes en la educación matemática.

Este desempeño matemático de los futuros profesionales, con la aplicación eficaz de las tecnologías computacionales, pude ser entendido como un componente didáctico y se inserta en una proyección educativa dentro de un contexto, como lo afirma Claro (2010) al decir que el valor instrumental de la práctica no está en las TIC, sino en cómo se integran en los procedimientos didácticos y se insertan en la dinámica de los métodos activos de forma articulada y da sentido en el desempeño investigativo de los futuros profesionales en sus actividades académicas dentro de un contexto determinado.

Por tal razón, el desempeño de los futuros profesionales, en la dinámica del proceso de enseñanzaaprendizaje de la matemática, tiene como propósito esencial formar un profesional capaz de sistematizar los conocimientos científicos y extraer propuestas, no solo de forma técnica, sino con el progreso de las tecnologías, en la integración de la teoría con la práctica para la solución de problemas que enfrenta el futuro profesional, lo cual le permite adaptarse a los futuros cambios, en su proceso formativo.

Se asume en el presente artículo, para la valoración de la dinámica de las tecnologías en la formación matemática, los criterios de Duhalde y González (1997) y Ortega y Francisco (2007), quienes reconocen que la principal motivación en la sistematización de las TIC, es promover en los futuros profesionales un razonamiento lo cual les permita trascender sus limitaciones cognitivas al incluir ciertas operaciones lógicas para el logro de nexos afectivos con el proceso de la investigación científica.

Por tanto, se desarrolla un proceso de disponibilidad para aprender, lo cual se potencia a través de los procedimientos didácticos, al garantizar una adecuada, contextualización de los problemas. Por otro lado, se asume también los criterios de Wongo, Faustino y Pérez, (2013) que plantean:

(...) los insuficientes profesionales de matemática en la sociedad angoleña, poco se interesan con la contextualización de los problemas e interpretación de los resultados, recorriendo a los aspectos de su profesión, por formas a elevar los niveles de comprensión en matemática y atracción de los futuros profesionales hacia las investigaciones (p. 488).

No obstante, en la práctica, el nivel de conocimiento matemático aplicado en las tecnologías computacionales y la motivación, que arriban los futuros profesionales angoleños en las diferentes carreras universitarias es alarmante. Es necesario también desarrollar un proceso de formación que tenga en cuenta el vínculo directo entre la técnica y las tecnologías actualizadas para la dinámica del proceso de enseñanzaaprendizaje de la matemática.

Las debilidades epistemológicas se notan en su desempeño investigativo aplicado a las tecnologías computacionales y esto repercutirá negativamente en un futuro próximo. Por tanto, para alcanzar el progreso científico de alto nivel que contribuya a superar la actual situación alarmante en la sociedad angoleña, es necesario llevar a los futuros profesionales a la creatividad mediante la sistematización de las TIC.

Mediante la sistematización de las TIC, se lleva a la creatividad de los futuros profesionales aplicando las técnicas gráficas de trabajar con problemas contextualizados en ambientes virtuales y permite presentar la información matemática (gestión del ambiente virtual), que desarrolla el pensamiento investigativo cuando se procesa la nueva información a través de la generación de los contenidos matemáticos. De ahí que, la creatividad mediante la sistematización de las TIC, revela patrones, interrelaciones e interdependencias cuando las herramientas tecnológicas son aplicadas de forma adecuada y también estimulan el pensamiento creativo si se orienta la atención del futuro profesional hacia estos patrones, se les induce a razonar y deducir sobre las características de los fenómenos sociales, que carecen de modelos matemáticos para su solución. (Figura 3). 
Figura 3: Creatividad mediante la sistematización de las TIC

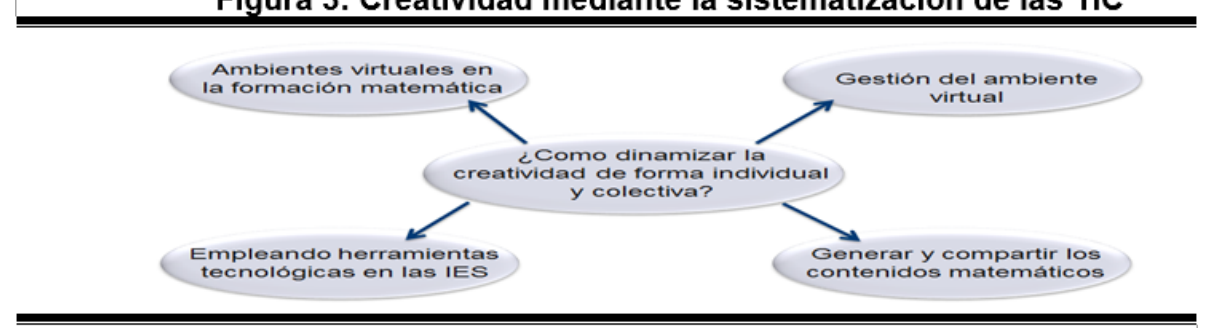

Fuente: Elaboración propia (2018)

FIGURA 3

Creatividad mediante la sistematización de las TIC

Fuente: Elaboración propia (2018)

Por otro lado, la política educacional superior angoleña deja bien claro qué trascendental son las TIC en la dinámica de la formación matemática. Los universitarios que ingresan en las instituciones de enseñanza superior, se convierten en un profesional no solamente de título, sino con la habilidad para desempeñarse en la sociedad. De ahí la necesidad que desde las clases se contribuya al fortalecimiento de su razonamiento lógico, siendo imprescindible para ellos darle suma importancia a las potencialidades de las tecnologías en sus investigaciones.

Por tanto, para el logro del desempeño hacia la formación matemática es necesario crear en los futuros profesionales las habilidades para aplicar las tecnologías en sus investigaciones, la cual permitirá actualizar los conocimientos como consecuencia de los cambios, en los avances de la tecnología y de las ciencias al desarrollo de sus investigaciones, buscando solución a los problemas profesionales lo que significa desarrollar el proceso investigativo y lograr resultados que respondan desde la particularidad de la carrera, a las diversas áreas del saber desde una integración interdisciplinaria hasta las necesidades que se manifiestan en la sociedad actual.

De este modo, es innegable la importancia y necesidad de las TIC, para dinamizar el proceso de enseñanzaaprendizaje de la matemática y su desarrollo en la sociedad. Sin embargo, en las IES angoleñas, no siempre se aprovecha al máximo el potencial de estas tecnologías, pues en el proceso de incorporación e integración que se lleva a cabo, no siempre se toman en cuenta los aspectos contextualizados a la realidad angoleña, cuestión que atenta con la calidad de formación del universitario.

\section{Procedimientos Metodológicos}

Análisis empírico de la situación actual del proceso de formación matemática en las IES con el uso de las TIC.

El enfoque experimental del presente artículo, que se implementó para detectar las limitaciones epistemológicas actuales en la utilización de las tecnologías computacionales en la carrera de Licenciatura en Educación Matemática, específicamente en las provincias de Bié, Huambo, Lubango y Luanda fue la recolección de datos cuali-cuantitativos para diagnosticar la situación actual que incluye cuestionarios, entrevistas y observaciones a clases en actividades investigativas desarrolladas por los alumnos-alumnas, con una edad comprendida entre los 20 y 34 años a través de la asignatura Ecuaciones Diferenciales e Integrales.

Antes de diagnosticar la situación actual del estudiantado, se determinó la confiabilidad de los instrumentos (cuestionarios, entrevistas y la guía de observación) mediante su aplicación inmediata a 10 alumnos y 10 alumnas de la carrera de Licenciatura en Educación Matemática de las IES en las provincias de Bié, Huambo para calcular el coeficiente Alfa de Cronbach, arrojando un valor de 0,9596 y permitió asegurar que los instrumentos tienen un $95,96 \%$ de fiabilidad, considerado aceptable para seguir con las investigaciones en otras instituciones (Figura 4). 
Figura 4: criterios emitidos por los jueces

\begin{tabular}{ll}
\hline \hline \multicolumn{3}{l}{ Estadísticos de contraste para los jueces desde 2013 hasta 2018 } \\
\hline \hline $\mathrm{N}$ & 23 \\
\hline $\mathrm{W}$ de Kendalla & 0,157 \\
\hline Chi-cuadrado & 51,095 \\
\hline Gl| & 13 \\
\hline Alfa de Cronbach. & 0,9596 \\
\hline \hline
\end{tabular}

a. Coeficiente de concordancia de Kendall de los jueces encuestados desde 2013 hasta 2018

Fuente: Elaboración propia del colectivo de autores (2016).

\section{FIGURA 4}

criterios emitidos por los jueces

Fuente: Elaboración propia del colectivo de autores (2016).

Los cuestionarios, entrevistas y la guía de observaciones a clases fueron distribuidos bajo la previa autorización y coordinación de los directivos de la IES en las provincias de Bié, Huambo, Lubango y Luanda, se estableció indicadores y subindicadores con preguntas abiertas ajustadas al estilo Likert, que consistió en asignar a través de una escala valorativa de uno a cinco el nivel de conocimiento que posee sobre la metodología utilizada para el uso de las tecnologías computacionales en la dinámica del proceso de enseñanzaaprendizaje de la asignatura Ecuaciones Diferenciales e Integrales, así los logros y limitaciones percibidas como parte de estos procesos.

El análisis empírico se realizó mediante el desarrollo del diagnóstico de la situación actual sobre el uso de las tecnologías computacionales en una muestra constituida por 30 profesionales y 150 futuros profesionales de la carrera de Licenciatura en Educación Matemática durante el año lectivo de 2013 hasta 2018 lo que permitió conformar dos grupos de análisis para la experimentación y el control, a fin de identificar las limitaciones epistemológicas en la aplicación de las tecnologías computacionales en la dinámica del proceso de enseñanza-aprendizaje de las ecuaciones diferenciales e integrales.

Se orientó a la población participante de futuros profesionales en tareas concebidas como problemas profesionales relacionados con el análisis e interpretación de los resultados que se manifiestan en la apropiación de los conocimientos sobre ecuaciones diferenciales e integrales, con el propósito de aplicar asistentes matemáticos, en situaciones sociales. Se realizó la revisión en la actividad de los profesionales para determinar el nivel de preparación que poseen en el uso de las tecnologías computacionales y resolver problemas profesionales relacionados con el análisis e interpretación de los resultados de las ecuaciones diferenciales e integrales, estableciéndose los siguientes indicadores y subindicadores:

Primer indicador: Interpretación de los resultados de las ecuaciones diferenciales e integrales con el uso de las tecnologías computacionales.

Subindicadores:

a) Determinación en la observación del tipo de problema a resolver desde las tecnologías computacionales que subyacen en la formulación de la problemática.

b) Delimitación en la comprensión de los fenómenos, en correspondencia con el objeto de la profesión donde se manifiesten las ecuaciones diferenciales e integrales en el contexto social y el propósito a alcanzar con la aplicación de las tecnologías computacionales.

Segundo indicador: Obtención de la información necesaria sobre las ecuaciones diferenciales e integrales para plantear el problema en el contexto social con la ayuda de las tecnologias computacionales.

Subindicadores: 
a) Aplicación del conocimiento de las tecnologías computacionales, en el modo de actuación de los profesionales, para determinar las circunstancias, en que se manifiestan las variables a observar en la investigación sobre las ecuaciones diferenciales e integrales.

b) Empleo de las fuentes de información, en correspondencia con los fenómenos que aportan las variables en investigación para solución de las ecuaciones diferenciales e integrales.

Tercer indicador: Significación del empleo de las tecnologias computacionales en las ecuaciones diferenciales e integrales como un problema profesional.

Subindicadores:

a) Transcendencia del tipo de análisis a realizar en correspondencia con el problema a resolver ya precisado.

b) Alcance a medir del impacto de las ecuaciones diferenciales e integrales, en correspondencia con las características de la problemática y el conocimiento del objeto de la profesión en que se manifiesta.

Cuarto indicador: Proceso de solución de las ecuaciones diferenciales e integrales modeladas con la utilización de las tecnologías computacionales.

Subindicadores:

a) Aplicación de los procedimientos adecuados, correspondientes al uso de las TIC en las ecuaciones diferenciales e integrales.

b) Interpretación del resultado obtenido de la aplicación de las tecnologías computacionales en el contexto profesional donde se plantea los conocimientos sobre ecuaciones diferenciales e integrales.

Los procedimientos metodológicos utilizados para la sistematización de los instrumentos de la observación partícipe, la entrevista profunda y la aplicación sucesiva de los cuestionarios en las clases, desde el 2013 hasta el 2018 para la apropiación y manejo de las tecnologías computacionales en la dinámica del proceso de enseñanza-aprendizaje de las ecuaciones diferenciales e integrales, posibilitó revelar que las formas organizativas se estructuran de manera más adecuada al proceso de enseñanza-aprendizaje de la nueva disciplina, sobre todo en lo relativo a la actividad académica donde más se ha trabajado en el desarrollo del pensamiento investigativo.

El 94\% de los profesionales presentan limitaciones metodológicas para la determinación del tipo de problema a resolver, aplicando la tecnología computacional Wolfram Mathematica. Mientras que el 92\% de los futuros profesionales, demuestran resultados muy bajos en los dos primeros subindicadores planteados anteriormente lo cual revela que los profesionales y futuros profesionales de la carrera de Licenciatura en Educación Matemática en las IES en las provincias de Bié, Huambo, Lubango y Luanda, aún carecen de técnicas apropiadas en el manejo eficiente de las TIC, para identificar los conceptos equivocados en el proceso de solución de las ecuaciones diferenciales e integrales.

En el año 2016, se aplicó cuestionarios a docentes y estudiantes de la carrera de Licenciatura en Educación Matemática, a fin de saber la eficiencia en la obtención de la información necesaria para plantear la transformada de Laplace, con la ayuda de las tecnologías computacionales. Sin embargo, el 89\% de los futuros profesionales cuestionados manifiestan, que la falta de independencia en la determinación del tipo de problemática la cual subyace en la situación para reforzar la comprensión del impacto de las ecuaciones diferenciales e integrarles en el contexto social, dificulta sus investigaciones.

El 80\% de los entrevistados, con más diez años de experiencia declaran que, dada la carencia de superación profesional se revelan insuficiencias en la aplicación de los procedimientos adecuados para emplear la serie de Fourier y sus aplicaciones en el contexto social, con el apoyo de la tecnología computacional Wolfram Mathematica, los cuales cuando necesitan, solicitan ayuda de especialistas para lograrlo, cuestión que dificulta el desarrollo de investigaciones en matemática. En entrevistas realizadas a profesionales, plantean que la 
asignatura Ecuaciones Diferenciales e Integrarles transitó desde una alta carga presencial a un número reducido de horas, esto hace que el proceso repose fundamentalmente en la actividad independiente de los futuros profesionales para una mayor apropiación de los conceptos. Pero se observó que la calidad de la promoción se vio afectada, así como las evaluaciones frecuentes y se detectaba en ellas una deficiente interpretación de conceptos.

El 77\% de los futuros profesionales entrevistados revelan insuficiencias en los procedimientos lógicos para solucionar las ecuaciones diferenciales ordinarias. Mientras que $82 \%$ no aprovechan la riqueza de la problemática en la proyección del análisis de las tecnologías computacionales para aclarar sus ideas, por la inadecuada selección de las variables a observar en el sistema de ecuaciones. Las entrevistas realizadas en los futuros profesionales, fundamentan que no logran asimilar grandes volúmene

El 77\% de los futuros profesionales entrevistados revelan insuficiencias en los procedimientos lógicos para solucionar las ecuaciones diferenciales ordinarias. Mientras que $82 \%$ no aprovechan la riqueza de la problemática en la proyección del análisis de las tecnologías computacionales para aclarar sus ideas, por la inadecuada selección de las variables a observar en el sistema de ecuaciones. Las entrevistas realizadas en los futuros profesionales, fundamentan que no logran asimilar grandes volúmenes de información y que los insuficientes folletos de la asignatura Ecuaciones Diferenciales e Integrales, no ayudan a la mejor comprensión de la explicación de los problemas planteados lo cual agudiza las limitaciones en el proceso de reconstrucción de los significados en la información matemática de los futuros profesionales.

En aras de minimizar las limitaciones epistemológicas en 2017 se aplicaron estrategias metodológicas para el perfeccionamiento de la dinámica del proceso de enseñanza-aprendizaje de las ecuaciones diferenciales e integrales usando las tecnologías computacionales y motivar a los futuros profesionales hacia el estudio de las carreras de Licenciatura en Educación Matemática. Se observó que el 60\% presentan algunas deficiencias en el empleo de tecnologías computacionales para viabilizar la dinámica del proceso de enseñanza-aprendizaje de las ecuaciones diferenciales e integrales.

En actividades investigativas desarrolladas desde el año 2017 hasta el 2018 por los grupos de experimentación y control a fin de reducir las deficiencias identificadas en el diagnóstico inicial, se empleó estrategias metodológicas que fortalecen la aplicación de las tecnologías computacionales, con problemas contextualizados en la dinámica del proceso de enseñanza-aprendizaje de las ecuaciones diferenciales e integrales lo que permitió constatar que el 30\% revelan ligeras limitaciones en la selección de los métodos adecuados para la interpretación de los resultados aplicando procedimientos en la solución de problemas contextualizados. Mientras que el 70\% revelan la necesidad de superar de forma permanente la utilización de las tecnologías computacionales en la dinámica del proceso de enseñanza-aprendizaje de las ecuaciones diferenciales e integrales en las IES, en las provincias de Bié, Huambo, Lubango y Luanda.

En su totalidad carecen de hábitos de estudio frecuente, porque sus niveles de abstracción e interpretación en la identificación de los conceptos de las ecuaciones diferenciales e integrales, no se encuentran ejercitados para reforzar la comprensión del contenido matemático, que en ocasiones completan su haber bajo la premisa de un conocimiento previo que aún es deficiente.

Todas estas insuficiencias están relacionadas con el grado de independencia para la solución de las ecuaciones diferenciales e integrales, modeladas con la utilización de las tecnologías computacionales y el dominio del contenido que deben emanar de un aprendizaje formativo. Es la resultante de un deficiente proceso de reconstrucción de significados, dado por las carencias de superación profesional en las tecnologías computacionales con problemas contextualizados que requiere de libros y medios tecnológicos adecuados, para incorporarlos a su dinámica y viabilizar la apropiación de contenidos esenciales de las ecuaciones diferenciales e integrales.

Métodos y procedimientos. La necesidad de mejorar la formación matemática con la aplicación de las tecnologías computacionales y analizar su repercusión en las IES, consistió en la caracterización de su estado 
actual, a través de encuestas aplicadas en los futuros profesionales y profesionales en las provincias de Bié, Huambo, Lubango y Luanda, en la República de Angola.

Se seleccionó de forma intencional durante la formación matemática desarrollada desde el año 2013 hasta el 2018, una muestra estratificada de 30 profesionales y 150 futuros profesionales de la carrera de Licenciatura en Educación Matemática, estructurada en un grupo experimental y de control. El 70\% de las personas seleccionadas corresponde al sexo femenino y 30\% al masculino, comprendidos entre los 35 a 50 años de edad. De 150 futuros profesionales seleccionados, $82 \%$ son mujeres y $18 \%$ hombres comprendidos entre los 20 a 45 años de edad.

Para evaluar el desempeño de los futuros profesionales en ambos grupos experimentales y de control, se aplicó la metodología cuali-cuantitativa ya usada por Roberto, Carlos y Pilar (2006) en el presente artículo para la corroboración de los resultados, desarrollada en el Centro de Estudios Educacionales de la Universidad de Ciego de Ávila, Cuba y su ejemplificación práctica en las IES en las provincias de Bié, Huambo, Lubango y Luanda en la República de Angola, en los alumnos-alumnas de la carrera de Licenciatura en Educación Matemática, del tercer año, sobre la interpretación de las limitaciones epistemológicas en el estudio de las tecnologías computacionales en la dinámica del proceso de formación matemática empleando el método histórico-lógico. Así como su factibilidad en la aplicación de una muestra constituida por dos grupos independiente de 15 profesionales titulares con un promedio de 34 años de experiencia y 75 futuros profesionales de ambos grupos de la carrera de Licenciatura en Educación Matemática, con la finalidad de valorar el desempeño de los universitarios en la aplicación de las TIC para contribuir al proceso de solución de las ecuaciones diferenciales e integrales, aplicando la técnica de muestreo aleatorio estratificado.

Para ambos grupos experimentales y de control, la evaluación de los procedimientos empleados se realizó mediante la aplicación de un diagnóstico de respuestas múltiples ajustadas al estilo Likert, lo cual consistió en asignar a través de una escala valorativa de uno a cinco, al espacio correspondiente a las respuestas adecuadas, según el nivel de conocimiento que posee sobre los procedimientos didácticos utilizados en el proceso de enseñanza-aprendizaje de las ecuaciones diferenciales e integrales, con el uso de las tecnologías computacionales en la solución de los problemas contextualizados para minimizar las limitaciones epistemológicas en los futuros profesionales de las Instituciones Superiores Pedagógicas en las provincias de Bié, Huambo, Lubango y Luanda.

\section{ANÁLISIS Y DISCUSIÓN DE LOS RESULTADOS DE LA PESQUISA}

Para la determinación de los posibles jueces se consideró la experiencia profesional vinculada a la temática que se investiga, la experiencia como docente e investigador, la categoría docente y científica e investigaciones realizadas que están relacionadas de alguna manera con las tecnologías computacionales al desarrollo del proceso de formación matemática, en las IES.

Sin embargo, autores como Da Ponte (2007), Faustino, Pérez-Sánchez y Craib (2016), Gungula y Faustino (2018), en sus investigaciones sobre las limitaciones en la formación matemática declaran que solo la mitad del profesorado tiene una investigación terminada, que pocas veces es el resultado del postgrado académico. Coincidiendo con estos criterios en la presente pesquisa se plantea que con frecuencia se declaran como resultados determinadas salidas de la investigación que no tienen el grado de terminación necesaria para su introducción en la formación matemática, lo cual limita su participación con prioridad en la transformación de las IES y el contexto social.

Por otro lado solo el $68 \%$ de los jueces encuestados poseen el grado científico de doctor en Ciencias Informáticas con la categoría docente de Profesor Titular (17), el 24\% son másteres que poseen la categoría de Profesor Auxiliar (5) y el 8\% son licenciados (1), que tienen vínculo directo con la disciplina principal integradora de la carrera de Licenciatura en Matemática, encargada del proceso de formación matemática de las y los alumnos a través de actividades matemáticas realizadas en la Universidad Máximo Gómez Báez, de 
Ciego de Ávila, Cuba y en las IES en las provincias de Bié, Huambo, Lubango y Luanda, quienes aseguran que las tecnologías computacionales pueden ser aplicadas de forma permanente, porque contribuyen a minimizar las imprecisiones en la dinámica del proceso de formación matemática de la carrera de Licenciatura en Educación Matemática de acuerdo con los criterios emitidos por los jueces.

Aunque se reconoció la pertinencia de la presente investigación, los jueces consideraron la necesidad de ofrecer un mayor nivel de concreción de las acciones específicas a seguir para una mejor orientación didáctica, así como explicitar más claramente las particularidades de los sistemas de ejercicios, ante la inquietud de una aplicación desacertada de la sistematización lógica de contenidos que no se corresponda con las características del modelo matemático planteado y evitar posibles cuestionamientos sobre la efectividad de la propuesta investigativa.

Para constatar los resultados que se aprecian en la dinámica del proceso de enseñanza-aprendizaje de las ecuaciones diferenciales e integrales, se observaron logros estadísticamente significados a los grupos experimentales y de control, se hicieron 14 observaciones (25 alumnos-alumnas) a clases en las IES en las provincias de Bié, Huambo, Lubango y Luanda en el año 2016, en las que se valoró la introducción de forma permanente de las tecnologías computacionales con problemas contextualizados y estos resultados fueron comparados con el diagnóstico inicial, a través de una prueba de hipótesis de los rangos con el signo de Wilcoxon para dos muestras relacionadas.

En ambos grupos de investigación, después del procesamiento de los datos, con el empleo del utilitario estadístico IBM SPSS Statistics 20, se obtuvieron los estadígrafos de posición y de dispersión, los cuales permitieron resumir la información que se comprueba con un impacto apreciable del uso de las tecnologías computacionales obteniéndose en la mayoría de los casos, diferencias altamente significativas (Figura 5).

Figura 5: Estadísticos descriptivos de las observaciones desde 2013 hasta 2018

\begin{tabular}{|l|l|l|l|l|l|l|l|l|}
\hline \hline Observaciones & $\mathrm{N}$ & Media & $\begin{array}{l}\text { Desviación } \\
\text { típica }\end{array}$ & & & & &
\end{tabular}

FIGURA 5

Estadísticos descriptivos de las observaciones desde 2013 hasta 2018

Fuente: Elaboración propia de los autores (2018)

Los resultados del análisis estadístico indican que la percepción de los futuros profesionales y profesionales, difiere significativamente en cuanto a los aspectos cuestionados y observados en las IES en las provincias de Bié, Huambo, Lubango y Luanda.

De este modo, los jueces seleccionados muestran un nivel de coincidencia en un $95 \%$ en sus respuestas en cuanto a la necesidad del perfeccionamiento de la dinámica de formación matemática con la implementación de las TIC, lo que demuestra que se minimizó las inconsistencias epistemológicas en la contextualización de los problemas en su proceso de formación, el hecho que el 95\% de los alumnos-alumnas en ambos grupos están interesados en continuar usando las tecnologías computacionales en el proceso de enseñanza-aprendizaje de 
las ecuaciones diferenciales e integrales, y solo el 5\% restante que realizó el diagnóstico final, entiende que es un poco complicado el uso de ellas en el proceso de solución de problemas.

\section{Conclusiones}

Como resultado de la valoración de los instrumentos aplicados para el diagnóstico realizado desde el año 2013 hasta el 2018, se buscó la comprobación de la validez y la fiabilidad de los resultados investigativos. La validez se refiere a aquella cualidad de los resultados de la investigación que llevan a aceptarlos como hechos incuestionables y la fiabilidad asegura que los resultados analíticos representan algo real e inequívoco en las insuficiencias manifestadas en las IES angoleñas. Así, los resultados obtenidos para el perfeccionamiento del proceso de formación matemática, con la aplicación de las TIC, se triangularon a través de sus distintas modalidades, las diferentes técnicas empleadas, las interpretaciones de los investigadores que apoyaron el estudio y las concepciones metodológicas ya sistematizadas por Rodríguez, Gil y García (2002), para contribuir a minimizar las limitaciones epistemológicas en la formación matemática.

En aras de contribuir a minimizar las insuficiencias epistemológicas en los futuros profesionales y viabilizar el desarrollo del proceso de formación matemática con aplicación de métodos dinámicos y tecnologías computacionales, en todas las IES angoleñas en la carrera de Licenciatura en Educación Matemática se puede concluir que:

El diagnóstico inicial y final realizado evidenció que aún se requiere continuar profundizando en estas metodologías, las cuales orientan la dinámica del proceso de enseñanza-aprendizaje de la matemática, sustentado en las tecnologías computacionales; a fin de superar en su totalidad las inconsistencias en la contextualización de los problemas en su proceso formativo contribuyendo al desempeño investigativo de los futuros profesionales en la sociedad angoleña para promover eficazmente la utilización de las TIC y la pedagogía de la matemática en un aprendizaje a largo plazo, dentro y fuera de las IES, de forma tal que su tratamiento contribuya al desarrollo de la concepción matemática como un instrumento imprescindible para la transformación del país.

Las y los alumnos de la carrera de Licenciatura en Educación Matemática, del tercer año, no se apropian de los contenidos matemáticos, por las insuficiencias en el proceso de enseñanza-aprendizaje de la disciplina Matemática General, dadas por la existencia de una contradicción entre la apropiación de contenidos y las exigencias formativas del futuro profesional.

La solución de las insuficiencias en la apropiación lógica de los contenidos, en correspondencia con el pensamiento investigativo limitó la pertinencia formativa del futuro profesional, lo cual implicó el desarrollo del proceso enseñanza-aprendizaje de la Matemática General teniendo en cuenta la relación dialéctica entre la lógica formal de esta disciplina y la lógica de apropiación de nuevos contenidos; que conllevó al desarrollo de la presente investigación como sustento de una estrategia de esta dinámica.

Se recomienda seguir profundizando sobre las tecnologías computacionales indispensables en el proceso de enseñanza-aprendizaje de la matemática, para suplir paulatinamente las deficiencias del aprendizaje y facilitar los procesos de interpretación que requiere de entornos virtuales, los cuales posibilitan la introducción de software al propiciar mejor desempeño investigativo de los futuros profesionales.

Desde este punto de vista y considerando que solo se logra la aplicación parcial de los resultados de la investigación en las IES, en las provincias de Bié, Huambo, Lubango y Luanda de la República de Angola, la aplicación de las tecnologías de la información y la comunicación, para mejorar la dinámica del proceso de formación matemática, se propone de manera acertada su perfeccionamiento, contextualizándolo en toda sociedad angoleña, bajo las condiciones y según las premisas mostradas en la presente investigación, lo cual avala un criterio más acabado del cumplimiento del propósito de esta y de la instrumentación sistemática de los resultados alcanzados en la pesquisa. 


\section{ReFERENCIAS}

Álvarez-Zayas, C. M. (1999). La escuela en la vida. Didáctica. La Habana: Editorial Pueblo y Educación.

Angola Pró-Reitoria para Reforma Curricular. (2007). Programas curriculares dos cursos de Bacharelato e Licenciatura. Luanda, Angola: Universidade Agostinho Neto.

Angola. (2012). Plano Nacional de Formação de Quadros 2013 - 2020: programa de aç̧ão 2013-2014. Angola: Ministerio do Ensino superior.

Angola. (2016). Lei de Bases do Sistema de Educação e Ensino. Lei número 17/16. Angola: Assembleia Nacional.

Calero, M. (2008). Constructivismo pedagógico. Teorías y aplicaciones básicas. México: Alfaomega Grupo Editor S. A. de C. V.

Claro, M. (2010). La incorporación de tecnologías digitales en educación. Modelos de identificación de buenas prácticas. Recuperado de https://goo.gl/G9n9NV

Cruz-Pichardo, I.M. y Puentes-Puente, A. (2012). Innovación educativa: Uso de las TIC en la enseñanza de la matemática básica. EDMETIC. Revista de Educación Mediática y TIC, 1(2), 127-145.

Da Ponte, J. P. (2007). Investigations and explorations in the mathematics classroom. The International Journal on Mathematics Education, 39(5-6), 419-430.

Diéguez, R. (2001). Un Modelo del proceso de solución de problemas matemáticos contextualizados en la matemática básica para la carrera de Agronomía. [Tesis no publicada de Doctorado en Ciencias Pedagógicas]. Centro de Estudios Manuel F. Gran, Santiago de Cuba.

Duhalde, M., y González, M. (1997). Encuentros cercanos con la matemática. Argentina: Editorial: Aique

Faustino, A. (2014). La formación del pensamiento matemático-investigativo en los futuros profesionales de la carrera de Licenciatura en Matemática [Tesis no publicada de Doctorado en Ciencias Pedagógicas] Centro de Estudios Manuel F. Gran, Santiago de Cuba.

Faustino, A. y Pérez, S. (2013). Utilización de las TIC en la enseñanza de la estadística en la Educación Superior Angolana. Revista de Ciencias Sociales, 11(2), 0-31.

Faustino, A. y Wongo, E. (febrero, 2014). Methodological guidance for Angolan mathematical Formation. Journal of Data Management and Computer Science, 1(1), 001-004. Recuperado de http://www.apexjournal.org

Faustino, A., Del Pozo, G. y Arrocha, O. (2014). El proceso de enseñanza-aprendizaje cooperativo en la formación matemática de los futuros profesionales universitarios. España: Editado por Servicios Académicos Internacionales para eumed.net. Recuperado en http://www.eumed.net/libros-gratis/2014/1383/index.htm

Faustino, A., Pérez-Sánchez, N. y Craib-Díaz, E. (2016). Habilidades intelectuales en la formación matemáticainvestigativa angoleña en el perfil Ingenieril. Revista Multiciencias, 16(1), 60-68.

Faustino, A., Pérez, N., Diéguez, R (2013). El pensamiento matemático-investigativo desde el enfoque científico tecnológico. Revista Multiciencias, 14(1), 80-87 Recuperado de https://goo.gl/tghqKG

Faustino, A., Pérez, N., Diéguez, R. (2014). Consecuencia de la formación matemática investigativa en la Educación Superior Angolana. Editorial Universitaria Félix Varela, 3(2), 10-45.

Faustino, A., Pérez-Sánchez, N. y Gungula, E. W. (2017). Formación del Pensamiento Complejo Ingenieril en la Universidad "Óscar Ribas". Telos Revista de Estudios Interdisciplinarios en Ciencias Sociales, 19(3), 523-544.

Faustino A., Wongo E. y Craib E. (2015a). El enfoque dialéctico en la formación matemática y su repercusión científico tecnológica en el contexto social. Revista de Investigación Social, 6 (14), 574-602

Faustino A., Wongo E. y Craib E. (2015b). Formación de conceptos matemáticos mediante herramientas informáticas en el perfil pedagógico. Revista Multiciencias, 15 (2), 226-237.

Freire, P. (1998). Pedagogia da atonomia. Saberes necessarios á prática educativa. Brasil: Editora Paz e Terra.

Frómeta-Quintana, E. (2007). El proceso de mediación semiótica en la formación de los profesionales de la educación. [Tesis de Doctorado en Ciencias Pedagógicas]. ISP “Frank País García”, Santiago de Cuba. 
Fuentes, H. (2009). La concepción cientifica holistica configuracional: una alternativa en la construcción del conocimiento cientifico, su aplicación en la formación de los profesionales de la Educación Superior en la contemporaneidad. [Tesis de Doctorado en Ciencias Pedagógicas] Centro de Estudios Manuel F. Gran, Santiago de Cuba.

Fullan, M (2001). The new meaning of educational change. Nueva York, EEUU: Teachers

Gómez, A. (19 de mayo de 2015). Contexto favorable para avanzar en el restablecimiento de relaciones. Granma, 1 .

Gungula, E. W. y Faustino, A. (2018). Dilema da formação matemática em Angola: falta de iniciativas próprias ou de compromisso com a qualidade de ensino? Revista Actualidades Investigativas en Educación, 18(3), 1-22. DOI: https://doi.org/10.15517/aie.v18i3.33459

Gungula, E. W., Dieguez-Batista, R. y Ugartemendía, E. P. (2015). Estrategia didáctica para el perfeccionamiento del proceso de formación interpretativa en la matemática superior. Revista Actualidades Investigativas en Educación, 15(2), 1-42. DOI: http://dx.doi.org/10.15517/aie.v15i2.18954

Kozma, R. (2008). Comparative analysis of policies for ICT in education. International handbook on information technology in education. Recuperado de https://goo.gl/Bc8Qik

Martínez, A. (1999). El estudio de casos como técnica didáctica -estudio de una experiencia en la enseñanza universitaria. Innovación educativa, (9), 25-53. Recuperado de https://goo.gl/iF3Qnx

Núñez, J. (2012). La Universidad y sus compromisos con el conocimiento, la Ciencia y la Tecnología. Revista Complutense de Educación, 20(2), 13-15.

Ortega R. y Francisco J. (julio - diciembre de 2007). Modelos didácticos para la enseñanza de las ciencias naturales. Revista Latinoamericana de Estudios Educativos, 3(2), 41 - 60. Recuperado de https://goo.gl/U6KLDU

Papanastasiou, E. y Ferdig, R. (2006), Computer Use and Mathematical Literacy: An Analysis of Existing and Potential Relationships. Journal of Computers in Mathematics and Science Teaching, 25(4), 361-371.

Pardo, M. E.: (2004). Las tecnologías de la información y las comunicaciones en la dinámica del proceso docente educativo en la educación superior. [Tesis no publicada de Doctorado en Ciencias Pedagógicas] Centro de Estudios Manuel F. Gran, Santiago de Cuba

Pérez, N. (2009). El proceso de formación investigativa sistematizada en la Educación Superior [Tesis no publicada de Doctorado en Ciencias Pedagógicas] Centro de Estudios Manuel F. Gran, Santiago de Cuba.

Quesada, A. (2010). Aprendizaje Colaborativo e Interuniversitario en Línea. [Tesis de Doctorado en Ciencias de Educación]. Universidade de Lisboa, Lisboa, Portugal.

Quitembo, A. D. J. (2010). A formação de professores de matemática no Instituto Superior de Ciências de Educação em Benguela - Angola. Um estudo sobre o seu desenvolvimento [Tesis de Doctorado en Ciencias de Educación]. Universidade de Lisboa, Lisboa, Portugal.

Ramírez, R. (2010). Transformar la universidad para transformar la sociedad. Quito-Ecuador: Senplades. Disponible en: https://goo.gl/w6CVLM

Ramírez, W. (2012). La experiencia de usuario y la usabilidad. SCHEMA, (1), 67-86. Recuperado de https://goo.gl/ BkP8J8

Rico, L. y Gil, M. (2003). Elaboración de una encues $\neg$ ta para el estudio de las creencias de los profesionales de matemáticas sobre Evaluación. En E. Filloy (Coord.). Matemática educativa aspectos de investigación actual (pp. 187-217). México D.F.: Fondo de Cultura Económica

Roberto S., Carlos C. y Pilar L. (2006). Metodología de la investigación. Perú: Editora El comercio S.A

Rodríguez, G., Gil, J. y García, E. (2002). Metodología de la investigación cualitativa. La Habana: Editorial Ciencias Sociales.

Santos, M. (1997). La transferencia del conocimiento y la formulación de problemas en el aprendizaje de las matemáticas. Revista Mexicana de Investigación Educativa, 2(3), 11-30.

Tünnermann, C. (2011). El constructivismo y el aprendizaje de los futuros profesionales. Universidades, 61 (48), 21-32. Recuperado de: http://www.redalyc.org/html/373/37319199005/

Vigotsky, S. (1989). Historia del desarrollo de las funcionespsíquicas superiores. La Habana: Editorial Científico Técnica. 
Wongo, E., Faustino, A. y Pérez E. (2013). El contexto angolano de formación matemática: un problema que se arrastra desde la base. Revista da Avaliação da Educação Superior, 18(2), 487-499 Recuperado de http:// www.redalyc.org/articulo.oa?id=219127939013

\section{Notas}

[1] Pensamiento investigativo, es un "procedimiento lógico, que viabiliza el proceso mental abstracto secuencial de razonamientos, juicios y procedimientos de búsqueda de información matemática” (Faustino; Pérez y Diéguez, 2013, p. 84).

[2] Innovar, en el presente artículo es, introducir técnicas al ámbito del proceso de enseñanza-aprendizaje, seguido de un proceso de difusión de los resultados.

\section{BY-NC-ND}

